\title{
Propuesta de mejora de procesos software basada en PSP para contextos de teletrabajo*
}

\author{
Fáber D. Giraldo** \\ Mónica M. Villegas ${ }^{* * *}$
}

\author{
Recibido: 12/12/2016 • Aceptado: 28/08/2018 \\ https://doi.org/10.22395/rium.v18n34a6
}

\begin{abstract}
Resumen
En este artículo se presenta una propuesta de especificación de mejora en el proceso software para contextos de teletrabajo basada en el framework Personal Software Process (PSP) del Software Engineering Institute (SEI). La propuesta describe una especificación de un proceso software, para que los desarrolladores que inician su trabajo en la modalidad de teletrabajo, y que no tengan este proceso definido, mejoren la forma de realizar los desarrollos. Tradicionalmente, un profesional de software, al incursionarse en el modo del teletrabajo, está ausente de un entorno laboral usual y se somete a sus propias maneras de llevar a cabo un desarrollo, por lo cual, se puede presentar que el profesional no tenga un proceso específico para desarrollar software o que no conozca alguno en particular. La propuesta en este artículo se enfoca en brindar una mejora para que pueda tener un proceso software base por el cual pueda abordar los retos particulares del teletrabajo.
\end{abstract}

Palabras clave: mejora de proceso software; teletrabajo; PSP; PSPT.

Proyecto final del espacio académico Aseguramiento de la Calidad de Procesos Software, Maestría en Ingeniería énfasis en Ingeniería de Software, Universidad del Quindío, Armenia, Colombia.

** PhD. en Informática Universidad Politécnica de Valencia (España). Docente de Planta - Investigador grupo Sinfoci, Universidad del Quindío, Armenia Colombia. Orcid: http://orcid.org/0000-0002-6111-3055 Correo electrónico: fdgiraldo@uniquindio.edu.co.

*** Candidata a Magister en Ingeniería Universidad del Quindío (Colombia). Investigadora Universidad Técnica Federico Santa María, Santiago, Chile. Correo electrónico: mmvillegasa@uqvirtual.edu.co. Orcid: https://orcid. org/0000-0001-9585-978X 


\title{
Software processes improvement proposal based on PSP for telecommuting contexts
}

\begin{abstract}
This article presents a proposal for improving the software process in telecommuting contexts based on the Personal Software Process (PSP) framework of the Software Engineering Institute (SEI). The proposal describes a specification of a software process for those developers who will begin their work on a telecommuting approach and doesn't have this process defined so they can improve the development of their projects. Traditionally, a software professional is absent of a usual work ambiance and is subjected to his ways of going through development, it is possible that he or she doesn't have a specific process for software developing or that he or she doesn't know of one in particular. This article suggestion is focused on giving an improvement based on software processes that allows the developer the engagement of the particular challenges of telecommuting.
\end{abstract}

Keywords: software process improvement; telecommuting; PSP; PSPT.

\section{Proposta de melhora de processos software baseada em PSP para contextos de teletrabalho}

Resumo

Neste artigo, é apresentada uma proposta de especificação de melhora no processo software para contextos de teletrabalho (ou home office) baseada no framework Personal Software Process (PSP) do Software Engineering Institute (SEI). A proposta descreve uma especificação de um processo software, para que os desenvolvedores que começam seu trabalho na modalidade de teletrabalho e que não tenham esse processo definido, melhorem a forma de realizar os projetos. Tradicionalmente, um profissional de software, ao entrar na modalidade de teletrabalho, está ausente de um contexto profissional usual e submete-se a suas próprias maneiras de realizar um desenvolvimento; portanto, pode ocorrer que ele não tenha um processo específico para desenvolver software ou que não conheça algum em particular. A proposta deste artigo está focada em oferecer uma melhora para que possa ter um processo software base pelo qual aborde os desafios particulares do teletrabalho.

Palavras-chave: melhora de processo software; home office; PSP; PSPT. 


\section{INTRODUCCIÓN}

Uno de los aspectos que se deberían tener en cuenta a la hora de trabajar en la modalidad de teletrabajo es la metodología y los procesos llevados a cabo, puesto que el entorno laboral y la forma en que se avanza y ejecuta el trabajo es diferente a la de un desarrollador de software trabajando en una empresa de modo presencial y convencional. Las metodologías de desarrollo de software, incluyendo las aproximaciones agilistas, promueven la interacción cara a cara como la mejor técnica de comunicación y afianzamiento de coherencia en un equipo de trabajo. En el teletrabajo, la dinámica y el control de trabajo es llevado a cabo, en gran parte, por el desarrollador de software a un nivel personal quien ejecuta sus propios métodos de control para el desarrollo de los proyectos.

En la actualidad, el teletrabajo genera un mayor impacto en Colombia, puesto que las empresas han estado incursionando en una modalidad que a nivel mundial se ha vuelto una tendencia en la forma de trabajo a distancia. Según el Ministerio de Tecnologías de la Información y las Comunicaciones (Mintic), actualmente se cuenta con aproximadamente 50.000 teletrabajadores en Colombia, y 280 organizaciones ya han firmado pacto [1] del teletrabajo con el Mintic [2].

Una de las claves para el éxito de la modalidad teletrabajo radica en el alto nivel de disciplina por parte de los trabajadores. Dicha disciplina está en función de las prácticas personales de desarrollo de software. Desafortunadamente, es muy frecuente que los desarrolladores en teletrabajo (tipo freelance) solo se preocupan por sus prácticas a nivel de implementación. Esto en parte es consecuencia de la ausencia de un marco metodológico que integre las prácticas de desarrollo de software en un entorno de teletrabajo.

Este artículo formula una propuesta metodológica para incorporar prácticas basadas en Personal Software Process (PSP) [3] en un entorno de teletrabajo. Con esta propuesta se pretende entregar un conjunto de prácticas disciplinadas que soporten efectivamente el teletrabajo aplicado en el desarrollo del software, a la vez que se promueve y consolida los esfuerzos gubernamentales alrededor de la formación en aseguramiento de la calidad de software usando la estrategia PSP del Software Engineering Institute (SEI).

Este trabajo está estructurado de la siguiente manera. La sección 1 presenta el método seguido para la formulación para la propuesta de proceso, incluyendo el soporte y motivación de la implantación del teletrabajo en Colombia, y el proceso software base sobre el cual se realizará una propuesta de mejora. La sección 2 presenta la especificación de la propuesta de mejora del proceso software orientado a contextos del teletrabajo basado en PSP. La sección 3 presenta una validación preliminar en 
cuanto a la implementación del proceso. Finalmente, se presentan las conclusiones y los agradecimientos.

\section{MATERIALES Y MÉTODOS}

El desarrollo de la propuesta planteada se basó en el proceso personal de software o por sus siglas en inglés Personal Software Process (PSP) y se le realizó una mejora para su aplicación en el teletrabajo. De esta forma, se inició con una revisión para determinar el estado actual de esta modalidad de labor en el país, se identificó el respectivo soporte a nivel de procesos software de las actividades normalmente consideradas en esta modalidad. Posteriormente, teniendo en cuenta los niveles y objetivos de calidad del framework PSP, se identifican las oportunidades de enriquecimiento metodológico del proceso para soportar las actividades de teletrabajo previamente identificadas. A continuación, se relaciona detalladamente la propuesta metodológica.

\subsection{Estado actual del teletrabajo en Colombia}

En Colombia, la modalidad de teletrabajo se ha venido adoptando por muchas empresas. Según el ministro TIC David Luna, para implementar la iniciativa del teletrabajo, el Mintic hizo alianza con el Ministerio del Trabajo con el propósito de establecer la guía y reglas con las cuales las empresas pueden implementar esta modalidad laboral. A dicho documento se le denominó el Libro blanco del teletrabajo [2]. Según el Mintic, entre las empresas que han estado implementando esta modalidad laboral se encuentran: Bancolombia, con 1.008 trabajadores y Bayer con 100. Entre otras compañías reconocidas, también se encuentran Carvajal, Nutresa, Pacific E\&P, Argos, Grupo M y la Cámara de Comercio de Bogotá [2].

Colombia ha mejorado la incursión de trabajadores en la modalidad laboral del teletrabajo, sin embargo, en Brasil y Argentina se registra una mayor cantidad de trabajadores bajo dicha modalidad, por ejemplo, Argentina registró un aumento de 320.000 oficinas en casa durante el 2004 a 2 millones en 2014 [2].

Según el consultor empresarial Luis Fernando Martín [2], las empresas tienden a contratar trabajadores en modalidad de teletrabajo, a causa de factores como:

- Reducción del espacio físico de trabajo.

- Disminución de costos operativos.

- Incremento de productividad.

- Contacto más directo con clientes.

- Mejor calidad de relación con el consumidor. 
Por dichos factores, la calidad de vida y la productividad de los empleados se ven mejoradas. Según el Mintic, al final del año 2014, el país contaba con un aproximado de 39.767 teletrabajadores, y según se estima, esta modalidad de trabajo creció un $26 \%$ desde que se firmó el decreto de la reglamentación del teletrabajo en el 2012 [4]. Según el Portal Teletrabajo en Colombia, se estima que en Colombia hay 50 mil teletrabajadores y se aspira a que en el 2018 la cifra alcance los 120 mil teletrabajadores en el país [5].

El teletrabajo en Colombia (promovido desde el Mintic) se ha venido rigiendo bajo los criterios expuestos por Jack Nilles (reconocido como fundador del teletrabajo). En teoría, los criterios de Nilles deberían ser adoptados por cualquier teletrabajador para laborar en modo teletrabajo correctamente. Los criterios de Nilles [6] se pueden resumir en:

1. Fuerte automotivación: el teletrabajador es más sensible a las distracciones y a la dispersión. Por otro lado, el aislamiento puede afectarlo psicológicamente. Por lo tanto, es necesaria la automotivación.

2. Autodisciplina: como el ambiente del teletrabajo no está sujeto al control físico y visual del ambiente tradicional de la oficina, la autodisciplina es fundamental.

3. Competencias y preparación: el candidato a teletrabajador tiene que tener las competencias necesarias para la actividad en cuestión y, además de eso, tiene que aprender a funcionar en este sistema.

4. Flexibilidad y espíritu de innovación: quien tenga dificultades en adaptarse a las nuevas situaciones, no es un buen candidato para el teletrabajo.

5. Formas de sociabilización: el teletrabajo coloca restricciones a la socialización. Son aconsejables soluciones mixtas (part-time en casa y en la oficina, o en régimen de centros de teletrabajo), o fomentar el encuentro entre teletrabajadores y clientes.

6. Régimen de voluntariado: nunca debe hacerse la selección por elección administrativa, todos los candidatos deben ser voluntarios y escogidos según criterios objetivos.

7. Ambiente familiar: la familia tiene que ser considerada en el análisis. Más aún, el teletrabajo no debe ser una mera estratagema para resolver problemas domésticos.

8. Combate las exageraciones: el ambiente de aislamiento puede generar dependencia en relación a la guía, alcoholismo y drogas. Hay que tener en consideración estos peligros.

9. Ambiente adecuado: tiene que haber un espacio claramente demarcado en el hogar para el teletrabajo. La separación en relación con las actividades familiares es crucial. 
10. Formación: entrene candidatos, los que los gerencian en las empresas y las familias de los teletrabajadores.

11. Sistema experimental: comience con un equipo de voluntarios, cree un sistema experimental y ensaye alternativas mixtas. Los test de funcionalidad del sistema deben, por lo menos, tener un año de duración.

12. Regla número uno: el teletrabajo no es ni un premio, ni un castigo. Es solo una manera diferente de trabajar.

Puesto que el teletrabajo está generando impacto en Colombia, el gobierno ha considerado la necesidad de implementar marcos legales y recomendaciones tanto para las empresas como para los empleados [7]. Entre las recomendaciones entregadas se promueve una buena gestión de proyectos, puesto que estos incluyen cronogramas, objetivos, metas, distribución de tareas, sistemas de comunicación y métodos de control. El uso de las tecnologías de la información en el teletrabajo, es un punto clave, puesto que sin las herramientas enmarcadas dentro de estas tecnologías no sería posible el teletrabajo.

Entre las diferentes herramientas que se pueden encontrar, se debe contar con algunas básicas, tales como herramientas para la comunicación ya sea por chat, video o voz, herramientas para el seguimiento de proyectos, herramientas para trabajo colaborativo, y sistemas de alojamiento para el control de la documentación; de igual forma, para el caso del software, las herramientas para el control de versiones es algo crucial, puesto que en el teletrabajo, está la opción de que los desarrolladores no se encuentren todos físicamente cerca, por lo que tener todo el software versionado, minimiza los riesgos de fallos y pérdidas durante los desarrollos.

A nivel jurídico, el teletrabajo en Colombia está soportado por la Organización Internacional del Trabajo (OIT), por la Ley 1221 de 2008 y el por Decreto 884 del 2012 que la reglamenta. Así, la Ley 1221 de 2008 establece el reconocimiento del teletrabajo en Colombia como modalidad laboral en sus formas de aplicación, las bases para la generación de una política pública de fomento al teletrabajo y una política pública de teletrabajo para la población vulnerable; crea, además, la Red Nacional de Fomento al Teletrabajo, con el fin de promover y difundir esta práctica en el país e incluye las garantías laborales, sindicales y de seguridad social para los teletrabajadores [8].

El Decreto 884 de 2012 especifica las condiciones laborales que rigen el teletrabajo en relación de dependencia, las relaciones entre empleadores y teletrabajadores, las obligaciones para entidades públicas y privadas, las administradora de riesgos laborales (ARL) y la Red de Fomento para el Teletrabajo. Así mismo, establece los principios de voluntariedad, igualdad y reversibilidad que aplican para el modelo [8]. 


\subsection{Proceso de software}

A la hora de desarrollar software, la calidad es uno de los atributos más importantes en un producto final, por lo que, desde su planeación, durante su desarrollo y al realizar la entrega final, se necesita de procesos específicos capaces de garantizar calidad, cumplimiento y organización. En esta situación es donde entran los procesos software.

Un proceso de desarrollo de software es un conjunto de personas, estructuras de organización, reglas, políticas, actividades y sus procedimientos, componentes de software, metodologías y herramientas utilizadas o creadas específicamente para definir, desarrollar, ofrecer un servicio, innovar y extender un producto de software [9].

La intención de un proceso de software apunta específicamente a modificar un proceso ya existente con el fin de mejorarlo o generar uno nuevo para cumplir y satisfacer requerimientos dados de una forma satisfactoria. Entre los elementos básicos de un proceso software están:

- Las actividades.

- El flujo de trabajo.

- Los roles que se desempeñan en la organización o equipo.

- El producto o artefacto que se obtiene como resultado del desarrollo.

Actualmente, existen diversos tipos de procesos software, por lo cual, se mencionarán algunos de ellos, que, de alguna forma, pueden ser utilizados para ser implementados en el teletrabajo, con el fin de tener un equipo más maduro y sólido. Entre dichos procesos, se encuentran:

- $\quad$ PSP (Personal Software Process) [3]

- TSP (Team Software Process) [10]

- $\quad$ RUP (Rational Unified Process) [11]

Se tomará como base el PSP, por lo cual se ahondará un poco más, el PSP (Personal Software Process), es un proceso que fue diseñado para que cada desarrollador de software pueda controlar y mejorar el trabajo desempeñado. Entre los objetivos del PSP, están:

- Lograr una disciplina de mejora continua en el proceso de desarrollo de software.

- Medir, estimar, planificar y controlar el proceso de desarrollo de software.

- Mejorar la calidad del proceso de desarrollo de software.

- Proveer calidad y productividad. 
La metodología del PSP maneja un conjunto de guiones que tienen como objetivo especificar los requerimientos de entrada, el proceso a seguir y los resultados que se esperan. La figura 1 resume la ruta metodológica de construcción de software propuesta por el framework PSP.

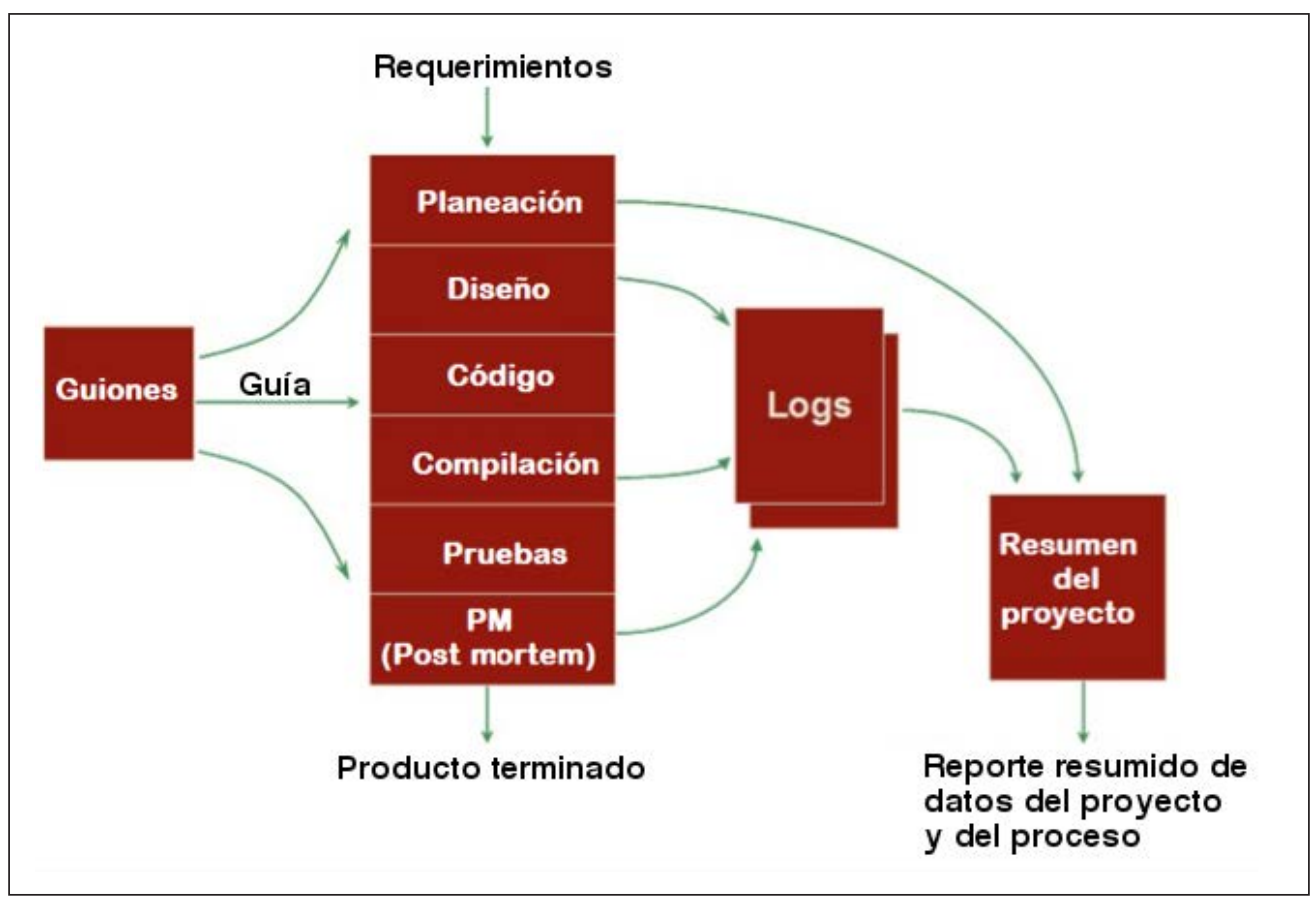

Figura 1. Elementos que provee el PSP

Fuente: traducida de [12].

La figura 2 presenta los niveles del framework PSP; cada uno de estos niveles tiene los siguientes objetivos:

- Objetivo para el nivel PSP0: establecer una línea base de rendimiento medido.

- Objetivo para el nivel PSP1: planear cronogramas, recursos y tamaños.

- Objetivo para el nivel PSP2: gestionar defectos y rendimiento.

- Objetivo para el nivel PSP3: escalar el PSP a proyectos de la vida real.

En Colombia, el Mintic ha venido realizando anualmente convocatorias para permitir a desarrolladores de software obtener certificaciones internacionales PSP y TSP [13]. El fin de dichas convocatorias es incentivar la certificación de profesionales que se desempeñan en el sector de las tecnologías de la información en este tipo de 
prácticas de procesos software, tradicionalmente consideradas como una estrategia metodológica orientada hacia el aseguramiento de la calidad de productos de software a partir del abordaje e intervención en procesos de desarrollo. La idea de las certificaciones es que los profesionales puedan ofrecer mejora continua y competitividad en las empresas de la industria de la tecnología, con el objetivo de que puedan implementar procesos adecuados para poder generar productos de alto nivel [14]. Sin embargo, cabe resaltar que el Mintic no proporciona espacios ni apoyos en los entornos laborales de los desarrolladores para realizar seguimiento o iniciar pilotos PSP, por lo cual, la aplicación de estos cursos oficiales patrocinados por el Mintic en entornos reales, es directamente dependiente al apoyo que reciban los desarrolladores formados en PSP por parte de las empresas a las que pertenecen.

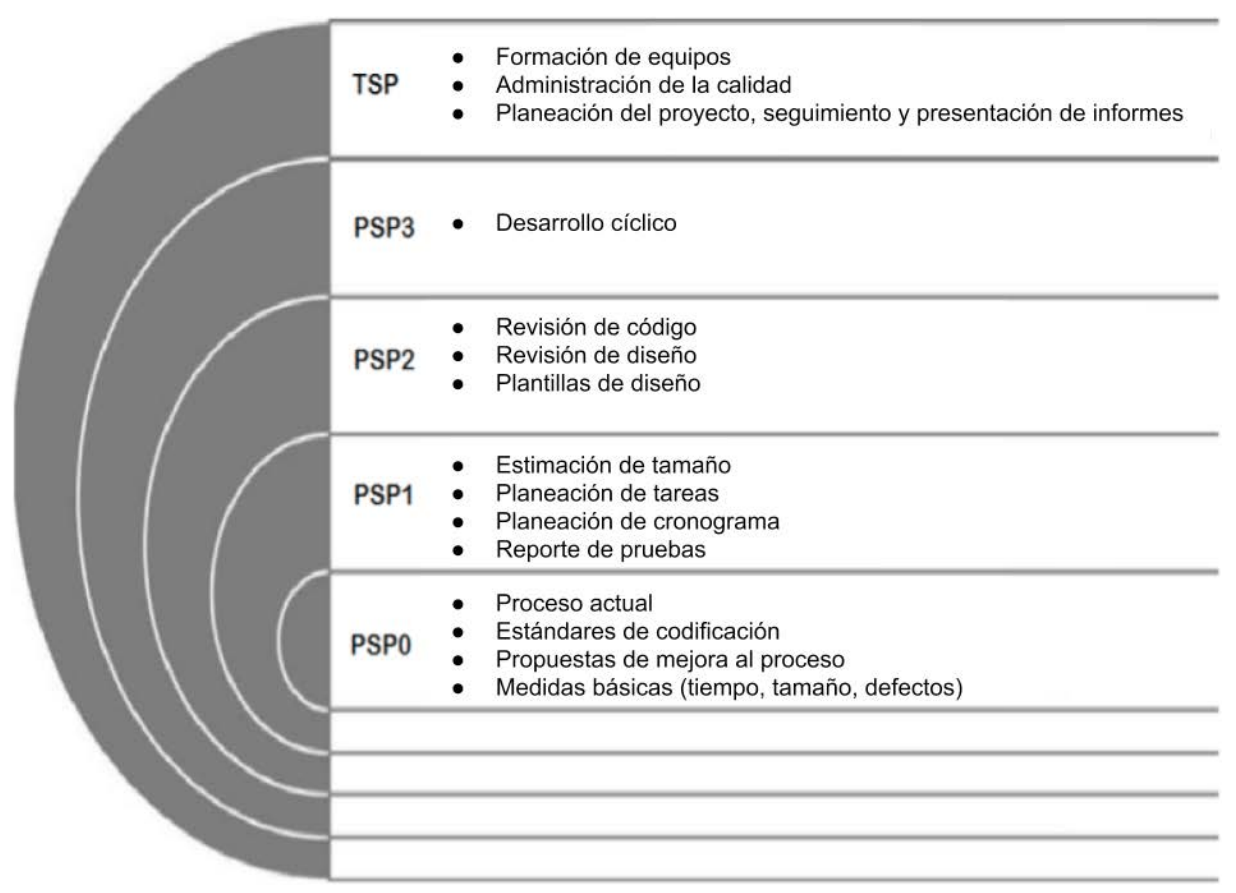

Figura 2. Procesos y objetivos del PSP

Fuente: traducida de [12]

\subsection{Estado del arte de procesos basados en PSP para teletrabajo}

De forma preliminar, al realizar una revisión en la literatura, se pudo encontrar la evidencia de un trabajo relacionado con el contexto de esta propuesta [15], en el cual los autores presentan unos requerimientos únicos para procesos software aplicados en 
ambientes de teletrabajo, enfocados en establecer procesos a nivel organizacional, de alineación, de recursos humanos, de infraestructura, de entrenamiento, de soporte, de documentación, de administración de la configuración, de administración de control al cambio y de administración de un sistema de soporte técnico, con el fin de establecer requerimientos para mejorar los procesos software aplicados en el teletrabajo.

\section{PROPUESTA}

Al realizar la revisión sobre el estado del teletrabajo en Colombia, de sus lineamientos, tanto procedimentales como judiciales, y de sus principios y objetivos; se pudo observar que en el teletrabajo no se puede solamente hablar de un proceso a nivel de software, sino que, en conjunto con este, se deberían tener en cuenta los otros factores que afectan a un empleado en modo teletrabajo. Específicamente, se deben tener en cuenta las reglas que estableció Jack Nilles, mencionadas en la sección 1.1.

La propuesta surge en base al mejoramiento del ritmo y la metodología de trabajo que lleva a cabo un profesional en modo teletrabajo en el área de desarrollo de software. La idea parte desde el hecho de que en esta modalidad existen diversos factores que pueden afectar el desempeño del trabajador $\mathrm{y}$, por lo tanto, reducir los buenos impactos que el teletrabajo en sí mismo por definición debe mejorar. El propósito es buscar una forma de mejorar la calidad del software, controlando los factores extra que se puedan presentar durante la ejecución de un desarrollo en la modalidad del teletrabajo.

Esta propuesta de mejora está enfocada en ser un punto de referencia para desarrolladores que no tienen implementados o no usan procesos software, y que, de alguna forma, se ha visto involucrada la calidad de sus productos por no tener un orden definido a la hora de llevar a cabo el desarrollo y, sobre todo, porque el ambiente en el que un empleado en modo teletrabajo pasa a trabajar, es muy diferente al de un empleado que está continuamente supervisado en una modalidad de trabajo convencional. Se especifica entonces a continuación la mejora del PSP enfocada al teletrabajo estableciendo que, por un lado, está el proceso involucrado con el teletrabajo y, por el otro, el PSP.

En lo que respecta al teletrabajo, se debe empezar, entonces, por establecer un horario de trabajo, el cual puede cambiar con el tiempo. Se recomienda estimar un horario de trabajo inicial para tener una referencia del tiempo que se invertirá en trabajar. Esto está relacionado con el hecho de que al estar en modo teletrabajo, se puede trabajar mucho más de lo que se debe ya que no se está en una oficina cumpliendo con un horario impuesto fijamente, y esto en algún momento puede implicar problemas de estrés en los trabajadores, lo cual puede influir en el desempeño de los mismos. También se recomienda escoger un horario de trabajo que permita al trabajador no tener cambios bruscos en su vida cotidiana, teniendo en cuenta que es una persona que 
debe tener relaciones sociales, familiares y otras actividades a parte del trabajo, esto se recomienda, de igual forma, para evitar problemas de estrés a largo plazo y evitar un mal desempeño a causa de esto.

Luego de establecer un horario de trabajo, se debe realizar un análisis sobre el lugar donde se trabajará. La modalidad de teletrabajo tiene la característica de que, si no es impuesto un lugar en particular por parte de la empresa, el teletrabajador puede escoger su lugar de trabajo, ya sea su casa, una oficina rentada, o algún otro lugar, algo que debe ser tomado muy en cuenta puesto que pueden haber factores de distracción, generadores de estrés y lugares no propicios ni idóneos para realizar un trabajo correctamente y por lo tanto, influir de igual forma, en el desempeño de los trabajadores. El análisis que se recomienda realizar al entorno o ambiente de trabajo, debería responder afirmativamente a las siguientes preguntas:

- ¿Se puede obtener una completa concentración en el ambiente o entorno laboral escogido?

- ¿El lugar escogido para laborar permite cumplir el horario de trabajo estimado?

- ¿El lugar escogido para laborar permite al trabajador estar cómodo y a gusto durante su horario de trabajo?

- ¿El lugar escogido para laborar tiene todos los elementos necesarios para trabajar?

Luego de contestar las preguntas anteriores, si todas fueron afirmativas, se podría considerar como un lugar adecuado para laborar, de lo contrario, si hubo respuestas negativas, se deben analizar los factores y verificar si se puede modificar para ser afirmativo, de lo contrario, se recomienda considerar otro lugar de trabajo que cumpla con una respuesta afirmativa para todas las preguntas expuestas. Es realmente importante contar con un ambiente laboral adecuado para poder obtener un buen desempeño.

Actualmente existen técnicas para analizar qué tan buena es la concentración de una persona al desarrollar software, y a su vez ir mejorando para administrar el tiempo, por lo cual, se recomienda hacer uso de este tipo de técnicas para hacer una autoevaluación y mejorar si es necesario, la concentración para desarrollar las tareas que se programen para la ejecución de los proyectos. Se recomienda la técnica de Pomodoro [16], puesto que es una de las técnicas más famosas alrededor de la administración del tiempo en el desarrollo de software, y que eventualmente, puede ayudar a mejorar la concentración del desarrollador.

En cuanto a la ejecución de tareas, se debe realizar un análisis de ejecución diario, el cual está basado en, inicialmente, tener una estimación de tareas diarias para realizar 
y poder llevar un control cotidiano. Para esto, se propone que al final del día se haga una comparación de las tareas estimadas para realizar con las tareas realizadas y poder saber exactamente qué problemas se tuvieron en cuanto a desempeño y ejecución del trabajo. El objetivo de esto es saber cómo corregirlos al siguiente día, puesto que la idea de este proceso es tener una mejora continua en cuanto al desempeño como teletrabajador. Se recomienda entonces el uso de la ecuación (1) con un umbral del $10 \%$ de diferencia de tareas:

$$
D T=\frac{(T E-T R) * 100 \%}{T E}
$$

Donde TE: tareas estimadas; TR: tareas realizadas; DT: diferencia de tareas. Se toma un DT de $10 \%$ como un porcentaje considerable, de lo contrario, si es mayor a este, se debe realizar un análisis del por qué no se cumplieron las tareas estimadas para ese día y cómo mejorar el desempeño ya sea a nivel técnico o por causas personales. La sugerencia de un DT del $10 \%$ se relaciona con el hecho de que, si un trabajador estima cierta cantidad de tareas al día, es porque desde su criterio las puede cumplir, algo que se va corrigiendo por medio del análisis de resultados diarios, por lo tanto, si estima cierta cantidad de tareas y solamente cumple algunas de ellas, pudo haber una mala estimación o tal vez algunos factores externos influyeron para que no se pudiesen llevar a cabo las tareas estimadas. Como ejemplo se describe el siguiente caso: un trabajador estima 5 tareas que va a realizar en el día, pero al final del día se dio cuenta de que solamente cumplió 4, realizando el cálculo con la fórmula especificada se tiene lo siguiente:

$$
\begin{gathered}
\mathrm{TE}=5 \quad \mathrm{TR}=4 \\
D T=\frac{(T E-T R) * 100 \%}{T E}=\frac{(5-4) * 100 \%}{5}=\frac{100 \%}{5}=20 \%
\end{gathered}
$$

El DT fue del $20 \%$, lo cual es mayor al umbral sugerido (10\%), por lo tanto, se debe realizar un análisis del por qué se falló en completar las tareas estimadas y poder realizar las correcciones correspondientes, ya sea por falta de conocimiento en el tema, por cuestiones externas, causas personales o por una mala estimación. Por otro lado, el $10 \%$ se sugiere, ya que tiene en cuenta mínimos retrasos en alguna de las tareas, lo cual permite ir corrigiendo las estimaciones y analizar problemas que afecten el desarrollo del trabajo, teniendo presente que puede que no haya perfección en cuanto a la ejecución en tiempo, de las tareas. 
Por último, se recomienda al finalizar cada semana, hacer un análisis de posibles influencias en el teletrabajo, el análisis consiste en responder una serie de preguntas, con el fin de analizar el proceso personal del teletrabajador, así se podrán verificar diversos factores como las distracciones, el ambiente laboral, elementos de trabajo, comunicación, automotivación y horario de trabajo. Las preguntas que se deberán responder al finalizar cada semana son las siguientes:

1. ¿Hubo distracciones?

2. ¿Si hubo distracciones, cuáles distracciones obstaculizaron el desempeño laboral?

3. ¿El ambiente laboral donde se trabajó fue adecuado?

4. ¿Si el ambiente laboral no fue adecuado, cuáles situaciones lo hicieron no adecuado?

5. ¿Se contó con todos los elementos de trabajo adecuados para llevar a cabo un buen trabajo?

6. ¿Si no se contó con todos los elementos de trabajo adecuados, cuáles elementos hicieron falta?

7. ¿La comunicación entre el teletrabajador y la empresa fue adecuada?

8. ¿Si la comunicación entre el teletrabajador y la empresa no fue adecuada, fue por causas técnicas o personales?

9. ¿Si fue por causas técnicas, cuáles fueron?

10. ¿Si fue por causas personales, cuáles fueron?

11. ¿Se trabajó horario adicional al inicialmente estimado?

12. ¿Si se trabajó horario extra, cuál fue la causa?

13. Determinar el nivel de automotivación del teletrabajador durante la semana en una escala de 1 a 10, donde 1 es una automotivación casi nula y 10 una automotivación completa.

14. Si la automotivación estuvo por debajo de 10, especificar el por qué la automotivación no es la mejor.

Estas preguntas serán importantes en cuanto al análisis semanal del desempeño del teletrabajador, puesto que ayudarán a entender problemas de desempeño y a tener un indicio de cuál es la causa de dichos problemas. Estas preguntas fueron establecidas a partir de los criterios de Nilles, ya que un teletrabajador con falta de automotivación, 
falta de autodisciplina, sin un ambiente adecuado y sin la formación y conocimientos correspondientes, puede tener demasiados problemas y tal vez fracasar en esta modalidad de trabajo (teletrabajo). En cuanto a lo que cada pregunta puede aportar en la detección de problemas, se especifica lo siguiente:

Las preguntas 1 y 2 permiten detectar distracciones, luego de ser identificadas, deberá buscarse alguna forma de eliminarlas o, en su defecto, mejorar la situación para que no sean un obstáculo en cuanto al rendimiento del teletrabajador. Las preguntas 3 y 4 podrán detectar problemas en el ambiente laboral de una forma constante por lo que, al haber problemas en dicho ambiente, se deberá buscar una forma de corregir las situaciones no adecuadas. Las preguntas 5 y 6 permiten detectar problemas con los elementos de trabajo, ya sea por falta de implementos técnicos (equipamiento) o por documentación. Las preguntas 7, 8, 9 y 10 permiten identificar problemas técnicos para la comunicación del teletrabajador con la empresa y otros compañeros de trabajo, y de igual forma, permiten detectar si hay problemas personales o de relación entre trabajadores que es un punto importante en la relación entre el trabajador, la empresa y todos los integrantes de la empresa. Las preguntas 11 y 12 apuntan a dificultades de sobrecarga laboral, de obstáculos en el trabajo, de malas estimaciones o de conocimientos insuficientes para realizar las tareas asignadas. Estas preguntas son realmente importantes, ya que, a largo plazo, si se trabaja siempre usando horarios extras, el trabajador puede llegar a sufrir problemas de estrés y con esto bajar su desempeño, por lo cual es importante encontrar las causas del uso de horario extra para realizar los proyectos asignados. Las preguntas 13 y 14 hablan del nivel de automotivación del teletrabajador, algo realmente fundamental, puesto que un trabajador desmotivado no rendirá lo suficiente. Con estas preguntas se podrán ir detectando las razones de la desmotivación en el trabajador para saber cómo encontrar una forma de corregir estas situaciones, con el fin de mejorar el desempeño.

Por el lado del desarrollo de los proyectos de software, se debe empezar por una definición de los proyectos: sus requerimientos, tiempos de entrega y asignación de responsables. En cuanto a la codificación, en caso de que no se cuente con alguna regla en general por parte de la empresa, se recomienda establecer métodos profesionales para el desarrollo de software, tales como el uso de patrones de diseño, codificación estandarizada y entendible, es decir, que el código sea comprensible para que futuros desarrolladores puedan tener referencias sólidas. De igual, forma la documentación del código desarrollado es esencial en cualquier proceso, por lo cual se recomienda documentar la mayor parte posible, como punto crucial durante la ejecución de los desarrollos.

Para empezar a ejecutar el proceso de mejora, se debe hacer una síntesis del proceso que será implementado a los desarrolladores, por parte de los directivos, con el fin de que todos los interesados estén involucrados y conozcan completamente el 
modelo o proceso que será utilizado. Durante la ejecución del proceso de mejora, se debe ir analizando cuáles beneficios se han tenido con respecto al proceso que se llevaba a cabo anteriormente, de tal forma, que se puedan implementar mejoras a la propuesta o descartar completamente su funcionamiento. Se propone entonces llevar una documentación detallada sobre los procesos anteriores y el proceso de mejora, teniendo columnas para comparar tiempos de entrega, calidad, testing y documentación. Durante la finalización del proceso de mejora, se recomienda hacer estudios rápidos en cuanto a la percepción y motivación de los desarrolladores, hacer un seguimiento continuo, puesto que el ambiente laboral de un desarrollador en modo teletrabajo puede influir en su desempeño de forma diferente al de un empleado común dentro de una empresa en modo presencial. Se propone, entonces, siempre al final de cada proceso, tomar como aprendizaje todo lo ocurrido durante el desarrollo, con el fin de poder identificar falencias en el proceso de mejora y poder corregir dichas falencias a tiempo para poder tener un proceso con un gran significado en el negocio.

A nivel de especificación del proceso, basado en una mejora al PSP enfocado al teletrabajo, se describe en la tabla 1 un marco de trabajo que define las etapas del proceso PSP mejorado para el teletrabajo, proceso al cual se le asigna el nombre PPST (Proceso Personal de Software para Teletrabajo).

Tabla 1. Marco de trabajo para el proceso propuesto

\begin{tabular}{|c|c|}
\hline Etapa & Descripción \\
\hline $\begin{array}{l}\text { PPST-D (proceso personal de software para } \\
\text { teletrabajo - etapa diaria) }\end{array}$ & $\begin{array}{l}\text { Esta etapa del proceso está basada en la mejora para el } \\
\text { teletrabajo, se debe realizar un análisis de ejecución diario. }\end{array}$ \\
\hline $\begin{array}{l}\text { PPST-S (proceso personal de software para } \\
\text { teletrabajo - etapa semanal) }\end{array}$ & $\begin{array}{l}\text { Esta etapa del proceso está basada en la mejora para el tele- } \\
\text { trabajo, se debe realizar un análisis semanal de influencias en } \\
\text { el teletrabajo. }\end{array}$ \\
\hline $\begin{array}{l}\text { PPST-0 (proceso personal de software para } \\
\text { teletrabajo - etapa } 0 \text { ) }\end{array}$ & $\begin{array}{l}\text { Esta etapa del proceso está basada en la mejora para el teletra- } \\
\text { bajo, se debe realizar la estimación de horario de trabajo y el } \\
\text { análisis del lugar de trabajo. }\end{array}$ \\
\hline $\begin{array}{l}\text { PPST-1 (proceso personal de software para } \\
\text { teletrabajo - etapa 1) }\end{array}$ & $\begin{array}{l}\text { Esta etapa está basada en el nivel } 0 \text { del PSP (PSP-0), junto con } \\
\text { la inclusión de las etapas PPST-D y PPST-S. }\end{array}$ \\
\hline $\begin{array}{l}\text { PPST-2 (proceso personal de software para } \\
\text { teletrabajo - etapa 2) }\end{array}$ & $\begin{array}{l}\text { Esta etapa está basada en el nivel } 1 \text { del PSP (PSP-1), junto con } \\
\text { la inclusión de las etapas PPST-D y PPST-S. }\end{array}$ \\
\hline $\begin{array}{l}\text { PPST-3 (proceso personal de software para } \\
\text { teletrabajo - etapa } 3 \text { ) }\end{array}$ & $\begin{array}{l}\text { Esta etapa está basada en el nivel } 2 \text { del PSP (PSP-2), junto con } \\
\text { la inclusión de las etapas PPST-D y PPST-S. }\end{array}$ \\
\hline $\begin{array}{l}\text { PPST-4 (proceso personal de software para } \\
\text { teletrabajo - etapa 4) }\end{array}$ & $\begin{array}{l}\text { Esta etapa está basada en el nivel } 3 \text { del PSP (PSP-3), junto con } \\
\text { la inclusión de las etapas PPST-D y PPST-S. }\end{array}$ \\
\hline
\end{tabular}


En la figura 3 se muestra la relación entre los niveles del PPST, dichas relaciones se hacen en base al PSP adicionando los niveles propuestos para el teletrabajo. Se debe iniciar con el nivel PPST-0, puesto que este marca el inicio para el desarrollo pues está relacionado directamente con aspectos de ambiente laboral y horario de trabajo. Se especifican luego los niveles PPST-D y PPST-S, los cuales se deberán ejecutar de forma diaria y de forma semanal respectivamente durante todo el proceso de desarrollo, por lo cual se relaciona con todos los niveles del proceso, y no se recomienda dejar de ejecutarlos durante la vida del proyecto. Estos dos niveles marcan un punto de autoevaluación para el mejoramiento continuo del desarrollador, por lo cual es demasiado importante llevarlos a cabo durante todo el proceso. Los niveles PPST-1 al PPST-4 están basados en los niveles del PSP, y responden a los objetivos especificados para el PSP.

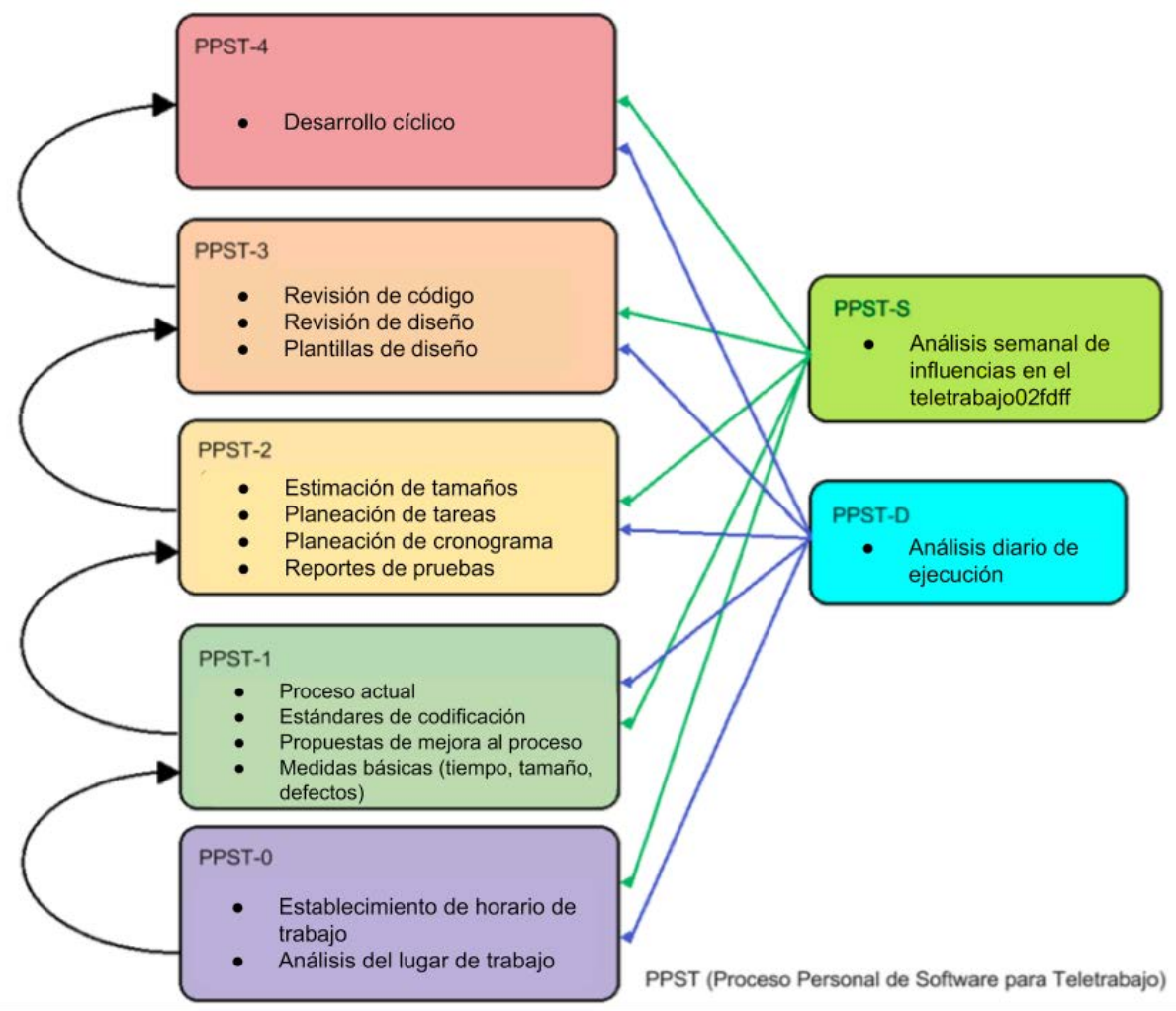

Figura 3. Relación entre las etapas del PPST

Fuente: elaboración propia.

El objetivo principal del marco de trabajo especificado en la tabla 1 y en la figura 3, es mejorar el PSP para el teletrabajo, tomando en cuenta las recomendaciones previamente descritas y siguiendo un orden específico, el cual está dado por el 
establecimiento de características específicas del teletrabajo (como horario y lugar de trabajo), las etapas propias del PSP y los análisis diarios y semanales que permiten controlar la forma de trabajo en el modo especificado y, a su vez, permiten detectar problemas específicos en cuanto al entorno y ejecución del trabajo.

\section{VALIDACIÓN PRELIMINAR}

Para realizar una validación preliminar, se hizo una socialización con teletrabajadores de un entorno real de desarrollo de software de la ciudad de Armenia (departamento del Quindío, Colombia). Al realizar la socialización de la propuesta, se obtuvo una respuesta positiva, puesto que hubo un consenso alrededor de la presencia en el teletrabajo de factores externos no relacionados directamente con el desarrollo, que pueden afectar el rendimiento de una persona al trabajar en esta modalidad. También se concluyó que el PSPT abarca aspectos que se deben revisar detalladamente (tal como se especifica) para poder minimizar efectos negativos en esta modalidad de trabajo. Además, se obtuvieron aportes por parte de los teletrabajadores consultados, en el sentido de que exponen el hecho de que sería importante tener en cuenta aspectos a nivel de salud ocupacional que no son tomados en cuenta por parte de las personas que se inician en esta modalidad de trabajo y que, de alguna forma, a futuro pueden existir aspectos que afecten la salud del trabajador. Entre los factores que fueron expuestos, está el hecho de tener en cuenta la posición de trabajo en un escritorio, el uso del mouse de la computadora para evitar problemas en las articulaciones de los brazos, y pausas constantes para evitar problemas de circulación de la sangre en el cuerpo.

A partir de la retroalimentación obtenida de la socialización, se espera que un teletrabajador pueda tener un proceso que involucre tanto aspectos de desarrollo, como aspectos externos que influyen en ese desarrollo sobre esta modalidad de trabajo.

\section{CONCLUSIONES}

El teletrabajo no solamente implica trabajar a distancia, puesto que hay varios factores involucrados tanto en la parte profesional del empleado, como en la parte personal. Hay que tener en cuenta todos estos factores, ya que, de alguna u otra forma, afectan a los teletrabajadores de una manera más directa que quienes trabajan presencialmente en una empresa de forma convencional.

Tener un proceso software definido en una empresa es algo crucial si se quiere obtener buena calidad en el software desarrollado. De igual forma, un proceso definido permite una organización entre los desarrolladores y los líderes, un entendimiento claro de lo que se tiene que hacer y quién tiene que hacerlo, y poder definir planes de mejora durante la vida de las empresas, siempre con enfoque en mejorar la calidad de 
los productos.

Cuando un trabajador inicia su camino laboral en modo teletrabajo, específicamente en el área del desarrollo de software, se puede presentar que no tenga lineamientos ni reglas para llevar a cabo el trabajo, por lo cual es importante establecer de forma mínima, un proceso de software que pueda brindarle al desarrollador reglas claras y básicas para llevar a cabo un trabajo ordenado y productivo.

En este artículo se formula una propuesta metodológica para extrapolar los principios del Personal Software Process (PSP) a los contextos emergentes de teletrabajo en el sector de la construcción de software en Colombia. A partir del proceso software formulado (PSPT) se derivan trabajos futuros orientados a validar la propuesta metodológica con mayor nivel de formalidad, considerando un protocolo de experimentación en ingeniería de software [17], aplicación de técnicas de estadística descriptiva, y la ejecución de procedimientos tipo casos de estudio (case study) [18] que permitan experimentar la propuesta en entornos controlados de desarrollo de software bajo la modalidad de teletrabajo.

\section{AGRADECIMIENTOS}

Mónica M. Villegas agradece al docente Fáber D. Giraldo por brindar sus conocimientos en procesos de software, los cuales fueron cruciales para el desarrollo de la presente propuesta. Este trabajo ha sido patrocinado por el Comité Central de Investigaciones de la Universidad del Quindío a través de la convocatoria interna para apoyos a estudiantes de maestría, modalidad investigación para el semestre 2016 II.

\section{REFERENCIAS}

[1] MinTIC, "Protocolo Pacto Teletrabajo," 2016.

[2] M. Echeverri, “La opción del teletrabajo ya suma 280 empresas y 50.000 empleados," en La República, 2016.

[3] M. Pomeroy-Huff et al., "The Personal Software Process (PSP) Body of Knowledge, Version 2.0," Software Engineering Institute, 2009.

[4] F. Cristancho-Dueñas, "Las cifras del teletrabajo en Colombia," [En línea], Disponible: https://blog.acsendo.com/las-cifras-del-teletrabajo-en-colombia/, 2016.

[5] Portal Teletrabajo Colombia, "Colombia avanza con paso firme en teletrabajo," [En línea], Disponible: http://www.teletrabajo.gov.co/622/w3-article-14760.html, 2018.

[6] Fedesoft, "El teletrabajo tiene su ciencia," [En línea], Disponible: http://fedesoft.org/noticiasfedesoft/el-Teletrabajo-tiene-su-ciencia/, 2016.

[7] P. T. Colombia. (2018). Gestión de proyectos a distancia: Consejos y Recomendaciones. 
Disponible: http://www.Teletrabajo.gov.co/622/w3-article-4464.html?_noredirect=1

[8] Portal Teletrabajo Colombia, “Marco jurídico,” [En línea], Disponible: http://teletrabajo.gov. co/622/w3-article-8098.html, 2018.

[9] M. Ruvalcaba, "Procesos de software," [En línea], Disponible: https://sg.com.mx/revista/1/ procesos-software, 2004.

[10] Software Engineering Institute, “TSP Overview,” [En línea], Disponible: https://resources. sei.cmu.edu/asset_files/Brochure/2010_015_001_72817.pdf, 2010.

[11] D. Gornik, "Rational Unified Process - Best Practices for Software Development Teams," [En línea], Disponible: http://user.it.uu.se/ hessel/project/RUP_bestpractices.pdf, 2001.

[12] W. S. Humphrey, PSP: A Self-Improvement Process for Software Engineers, Addison-Wesley Professional, 2005.

[13] Clearmodel. (2014). SEI-Certified PSP Developer: Frequently Asked Questions. Disponible: http://web.archive.org/web/20141129020408/http://seicertification.clearmodel.com/get-certified/software-development/become-an-sei-certified-psp-developer/ sei-certified-psp-developer-frequently-asked-questions/

[14] Icetex, "Fondo tsp/psp - cuarta convocatoria," [En línea], Disponible: https://www.icetex. gov.co/dnnpro5/es-co/fondos/fondosparaeldesarrollodeti/fondotsppsp.aspx, 2015.

[15] H. Guo, "Special requirements for software process improvement applied in teleworking environments," Presentado en Proceedings Second Asia-Pacific Conference on Quality Software, pp. 331-340, Hong Kong, 2001

[16] F. Cirillo, “The Pomodoro Technique,” [En línea], Disponible: https://francescocirillo.com/ pages/pomodoro-technique, 2009.

[17] C. Wohlin et al., Experimentation in Software Engineering. Berlín: Springer Publishing Company, 2012.

[18] S. Keng and M. Rossi, "Evaluation of information modeling methods-a review," Presentado en Proceedings of the Thirty-First Hawaii International Conference on System Sciences, pp. 314-322, vol.5, Hawaii, 1998. 